\title{
GINS2 attenuates the development of lung cancer by inhibiting the STAT signaling pathway
}

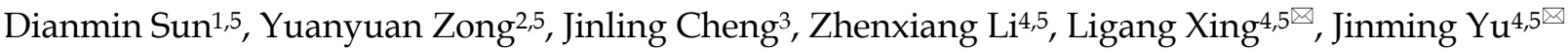 \\ 1. Department of Thoracic Surgery, Shandong Cancer Hospital Affiliated to Shandong University, Shandong Academy of Medical Sciences, Jinan, Shandong \\ 250117, China \\ 2. Department of Pathology, Shandong Provincial Hospital Affiliated to Shandong University, Jinan, Shandong 250117, China \\ 3. Department of Gastroenterology, Shandong Provincial Western Hospital, Jinan, Shandong 250117, China \\ 4. Department of Radiation Oncology, Shandong Cancer Hospital Affiliated to Shandong University, Shandong Academy of Medical Sciences, Jinan, \\ Shandong 272173, China \\ 5. Shandong University, Jinan, Shandong 250117, China
}

$\triangle$ Corresponding authors: Dr. Jinming Yu, Dr. Ligang Xing. Email: sdyujinming@163.com, xinglg@medmail.com.cn. No. 440, Jiyan Road, Shandong Cancer Hospital Affiliated to Shandong University, Jinna City, Shandong Province 250117, China. Tel: 86-0531-87984777; Fax: 86-0531-87984079

(1) The author(s). This is an open access article distributed under the terms of the Creative Commons Attribution License (https://creativecommons.org/licenses/by/4.0/). See http:/ /ivyspring.com/terms for full terms and conditions.

Received: 2020.04.07; Accepted: 2020.10.06; Published: 2021.01.01

\begin{abstract}
GINS complex subunit 2 (GINS2) controls DNA replication. GINS2 expression is upregulated in several kinds of aggressive tumors. However, the effect of GINS2 in lung cancer remains unclear. We performed TCGA database analysis to confirm the clinical significance of GINS2 in lung cancer. After silencing GINS2 in A549 cells, we performed MTT assays, flow cytometry assays, colony formation assays, cell cycle analyses and RNA sequence analysis to elucidate the effect of GINS2 on lung cancer. Moreover, we assessed tumor growth and analyzed body fluorescence in mice as a measure of tumor burden. The TCGA database analysis demonstrated that GINS2 mRNA and protein was highly expressed in three kinds of lung cancer tissues. Subsequently, knockdown of GINS2 inhibited cell proliferation, colony formation, cell cycle arrest and apoptosis in A549 cells. On the other hand, we also investigated the effect of GINS2 on tumor formation in vivo. The analysis of nude mouse tumors showed that the tumor volume and weight of shGINS2 mice were significantly smaller than those of the control mice. To reveal the mechanism of GINS2 in lung cancer, we collected A549 cells with GINS2 knockdown to examine the downstream gene expression changes. The results showed that STAT1 and STAT2 mRNA and protein expression were significantly upregulated after GINS2 knockdown in A549 cells. Our results suggest that GINS2 inhibits the proliferation of lung cancer cells by inhibiting the STAT signaling pathway, which may be a potential biomarker for the diagnosis or prognosis of lung cancer.
\end{abstract}

Key words: GINS2; STAT; lung cancer; proliferation; apoptosis

\section{Introduction}

Lung cancer is the leading cause of cancer-related death worldwide, and more than $85 \%$ of lung cancer patients suffer from non-small-cell lung cancer (NSCLC) [1,2]. Despite the improvement of treatment methods, the long-term survival rate of NSCLC remains low due to cancer recurrence and metastasis, with a 5 -year survival rate of less than $15 \%$ [3-5]. Therefore, new prognostic biomarkers and therapeutic targets need to be determined urgently.

GINS complex family members were named GINS complex subunit (GINS), including GINS2, GINS3 and GINS4. GINS complex family members play an essential role in the initiation of DNA replication and the cell cycle [6]. In addition, GINS2 may play an important role in cell division, specifically by promoting chromosome segregation [7]. It has been reported that GINS2 is involved in the tumorigenesis of various cancers, and its molecular weight is approximately $21 \mathrm{kDa}$ [8]. Specifically, GINS2 was found to be highly expressed in colorectal cancer by genome-wide gene expression profile analysis [9]. In addition, GINS2 is related to the invasive characteristics of breast cancer, and it is presumed that GINS2 is related to the metastasis of 
lung cancer [10]. Furthermore, the enhanced expression of GINS2 can promote the proliferation of leukemia cells and desensitize them to apoptosis [11]. These findings suggest that GINS2 may play a central role in tumorigenesis.

In the previous study, Chi et al discovered that knockdown of GINS2 inhibits proliferation and promotes apoptosis through the p53/growth arrest and DNA damage 45A pathway in NSCLC [12]. Additionally, another research group performed the experiment to demonstrate that GINS2 facilitates epithelial-to-mesenchymal transition in NSCLC cancer through modulating phosphoinositide-3kinase/protein kinase $\mathrm{B}$ and mitogen-activated protein kinase/extracellular signal-regulated kinase pathway [13]. In present study, we determined the clinical significance of GINS2 in lung cancer and inhibited the proliferation of lung cancer cells by inhibiting the signal transducer and activator of transcription (STAT) signaling pathway. In addition, we have gained a new insight into the important role of GINS2 in NSCLC development.

\section{Materials and Methods}

\section{Cell culture}

Human embryonic kidney 293 (HEK293), human MCF-7 breast cancer cells, human umbilical vein endothelial cell (HUVECs), human SW480 colon cancer cells, 95D, A549, NCI-H1299 and NCI-H1975 cells were purchased from American Type Culture Collection (ATCC) and grown adherently in a humidified incubator at $37^{\circ} \mathrm{C}$ with $5 \% \mathrm{CO}_{2}$. All cells were maintained in Dulbecco's modified Eagle's medium (DMEM) high glucose containing 10\% fetal bovine serum (FBS), penicillin $(100 \mathrm{U} / \mathrm{mL})$ and streptomycin $(100 \mathrm{mg} / \mathrm{mL})$. The medium was changed every two days.

\section{Construction of the GINS2 shRNA Lentiviral Vector}

The GINS2 shRNA vector was constructed with a lentiviral plasmid. The GINS2 (GeneBank accession number: NM_016095) and scrambled shRNA oligonucleotides are listed in supplementary table 1. They were inserted into pLentiHI plasmids at AgeI and EcoRI sites and confirmed by sequencing. The 8.9 packaging plasmid and VSVG envelope protein plasmid were combined with the shRNA empty vector and then cotransfected into HEK293T packaging cells using Lipofectamine 2000 (Invitrogen, USA) [14]. After transfection for $48 \mathrm{~h}$, the supernatant was collected and filtered through a $0.45 \mu \mathrm{m}$ filter to remove cellular debris to collect the virus particles.

A549 cells $\left(1 \times 10^{6} / \mathrm{mL}\right)$ were cultured in 6 well plates and infected with $4 \mu \mathrm{L}$ lentivirus. The virus titer was $5 \times 10^{8} \mathrm{TU} / \mathrm{mL}$. The expression of green fluorescent protein was observed after transfection for $72 \mathrm{~h}$ by fluorescence microscopy. The experiment was divided into two groups, shGINS2 and shCtrl.

\section{Animal models}

Eight-week-old male BALB/c nude mice were purchased from Beijing Vital River Laboratory Animal Technology Company and randomly divided into two groups, negative control (NC): GFP shRNA; and knockdown (KD): GINS2 shRNA, with 10 mice in each group. The protocol was performed according to a previous study [15]. We subcutaneously injected $5 \times 10^{6}$ A549 cells with shGINS2 or shCtrl lentivirus into the right flank of each mouse. Tumor size was measured every four days after injection, and then we calculated tumor volume according to the formula: volume $=$ length $\times$ width ${ }^{2} \times 3.16 / 6$. Animal care and experimental procedures were approved by the Animal Care and Use Committee of Shandong University.

\section{RNA extraction and real-time PCR}

We extracted total RNA from A549 cells or mouse tissues using RNAiso Plus (Invitrogen, USA) and immediately performed RT-PCR to synthesize cDNA using PrimeScript ${ }^{\mathrm{TM}}$ RT Master Mix (Takara, Japan). qRT-PCR was performed using specific primers and SYBR Green (Invitrogen, USA) on Applied Biosystems (Thermo, USA). qRT-PCR was performed in triplicate to amplify specific genes and normalize them to $\beta$-actin levels. Primer sequences are listed in supplementary table 2 .

\section{Differentially expressed gene analysis}

We performed the experiment according to a previous study [16]. First, clean reads were obtained from the results. Then, we aligned the clean reads with the human reference genome (GCF_000001405.38) using TopHat. Cufflinks was used to normalize the gene expression in RPKM (reads per million per kilobase). We used DESeq software to analyze the differentially expression genes to compare the $\mathrm{NC}$ and $\mathrm{KD}$ groups. The false discovery rate (FDR) was used to set the significance threshold of the $p$-value in multiple tests. The significance of gene expression was judged by FDR $\leq$ 0.05 and absolute value of fold change $\geq 2$ as the thresholds.

\section{Western blotting}

Western blotting was performed as previously described [17]. Briefly, cells were lysed and separated on $10 \%$ SDS-polyacrylamide gels. The following primary antibodies (Abs) were used: GINS2, B-cell 
lymphoma-2 (Bcl-2), Bax, caspase-3, cyclin dependent kinase inhibitor 1a (CDKN1A), CDKN1B, cyclin dependent kinases 4 (CDK4), cyclin D, bone morphogenetic protein receptor type 2 (BMPR2), interferon induced protein with tetratricopeptide repeats 1 (IFIT1), STAT1, STAT2, interferon regulatory factor 1 (IRF-1), tumor necrosis factor alpha induced protein 3 (TNFAIP3), interferon induced transmembrane protein 1 (IFITM1) and glyceraldehyde-3-phosphate dehydrogenase (GAPDH). Signals were detected using the Immobilon reagent (Millipore, USA) and visualized using a Bio-Rad ChemiDoc XRS (Bio-Rad, USA). Visualized signal intensities were quantitatively analyzed using Quantity One software (Bio-Rad, USA). All primary Abs were purchased from Cell Signaling Technology (Beverly, USA).

\section{Cell growth assay}

A549 cells were seeded into 96-well plates at a density of $5 \times 10^{3}$ cells/well. We transfected shCtrl or shGINS2 lentivirus into A549 cells during the logarithmic growth phase. We counted the cells daily using the Celigo Imaging Cytometer (Nexcelom Bioscience, USA) and repeated the experiment three times.

\section{MTT cell proliferation assay}

A549 cells were seeded into 96-well plates at a density of $5 \times 10^{3}$ cells/well, and cell viability was assessed using MTT (Thermo Fisher, USA). We added the MTT solution and incubated it for $4 \mathrm{~h}$ at $37^{\circ} \mathrm{C}$. Then, we added $10 \mu \mathrm{L}$ dimethyl sulfoxide to form formazan crystals and mixed for $3 \mathrm{~min}$. Then, we measured the absorbance at $490 \mathrm{~nm}$ by using a microplate reader (Tecan Infinite, Austria). The MTT assay was repeated three times.

\section{Colony formation assay}

A549 cells were seeded in six-well plates at $10^{5}$ cells/well, and the medium was changed every two days and cultured in $5 \% \mathrm{CO}_{2}, 37^{\circ} \mathrm{C}$. We transfected the lentivirus shGFP and shGINS2 into A549 cells and observed the status of the cells. After being washed with phosphate-buffered saline (PBS), cells were fixed with $4 \%$ paraformaldehyde for $30 \mathrm{~min}$. Then, the cells were stained with Giemsa staining solution for $8 \mathrm{~min}$ and washed 3 times with $\mathrm{ddH}_{2} \mathrm{O}$. We counted and photographed the colonies under a microscope (Nikon, Japan).

\section{Cell cycle analysis}

Flow cytometry analysis was used to detect cell cycle distribution. A549 cells were transfected with shGFP or shGINS2 lentivirus. Then, the cells were collected and washed with prechilled D-Hanks solution ( $\mathrm{pH} 7.2)$ and fixed for $1 \mathrm{~h}$ in prechilled $75 \%$ ethanol. Cells were stained with a solution containing D-Hanks solution, propidium iodide (Sigma, USA) and RNase concentrate $(10 \mathrm{mg} / \mathrm{mL})(1000: 25: 10)$ and analyzed on a flow cytometer (Guava ${ }^{\circledR}$ easyCyte Flow Cytometers, USA). Cell cycle analysis was performed in triplicate.

\section{Cell migration assay}

Cell migration was detected using a wound-induced migration assay [18]. In brief, A549 cells were seeded in 6 well plates at $1 \times 10^{5}$ cells/well. Once the cells grew and reached $80 \%$ confluence, a straight line was scraped with a pipette tip to create a "scratch", and the cells were washed 3 times with PBS. We replaced the medium with medium specific for the in vitro scratch assay. The wound healing images were captured at 0 and $48 \mathrm{~h}$ post-wounding under a Nikon microscope (Nikon, Japan). The ratio of the cell recovery area was measured to evaluate cell migration.

\section{Immunohistochemistry}

For the microscopic quantification of the GINS2 expression, the lung cancer tissue was cut into 8-10 sections as previously described [15]. To microscopic evaluation of GINS2 expression in the lung cancer tissues at different stages, serial paraffin sections of the lung cancer tissue were immunohistochemical (IHC) stained with the GINS2 antibodies (Sigma, USA). Secondary antibodies included anti-Goat IgG for GINS2 staining (Beijing Zhongshan Biotechnology, China).

Two independent observers blinded to the histopathological features and patient data of the samples evaluated and scored the degree of immunostaining. The scores were based on the proportion of positively stained tumor cells [graded as: negative ( $=0 \%$ positive), week ( $1-25 \%$ positive), positive $(26-50 \%$ positive) or strong positive $(>51 \%$ positive)] and staining intensity [categorized as 1 (no staining), 2 (weak staining, light yellow), 3 (moderate staining, yellow brown) or 4 (strong staining, brown)].

\section{The Cancer Genome Atlas (TCGA) data analysis}

GINS2 profiles of 515 lung adenocarcinoma tissues and 59 lung tissues were downloaded from TCGA database (http://genome-cancer.ucsc.edu) and used to determine the correlation among the GINS2 expression, clinicopathological features and prognosis of patients with lung adenocarcinoma.

\section{Statistical analysis}

The statistical analyses were carried out by one-way ANOVA followed by LSD test using 
GraphPad 7.0 software. In all cases, data were expressed as the mean \pm SEM. $P<0.05$ was considered statistically significant.

\section{Results}

\section{GINS2 was highly expressed in lung cancer specimens}

In a previous study, Han and his colleagues[19] performed RNA sequencing (RNA-Seq) to compare the transcriptomes of NSCLC and paracarcinoma tissue (PCT) to investigate gene expression. They enrolled 88 male patients (mean age, 61.2 years) with NSCLC, 54 patients with adenocarcinoma and 34 patients with squamous cell carcinoma. The heat map revealed that GINS2 was highly expressed in NSCLC patients (Figure 1A). Moreover, we compared GINS2 expression in lung squamous cell carcinoma (LSCC), large cell lung cancer (LCLC) and adenocarcinoma of the lung (ACL). The results showed that GINS2 was highly expressed in LSCC, LCLC and ACL patient lung tissues (Figure 1B, C and D).

In addition, we measured the mRNA and protein expression of GINS2 in LSCC, LCLC and ACL cancer tissues compared to PCT by RT-PCR and Western blotting. The results showed that GINS2
mRNA and protein expression were highly expressed in cancer tissue (Figure 2A, B, C and D), indicating that GINS2 was related to the occurrence and development of NSCLC.

The expression status of GINS2 was determined in patients with lung adenocarcinoma and the PCT by immunohistochemistry. GINS2 expression was not detected in PCT (Figure 2E). Of all the cancer specimens examined, $29(18.9 \%)$ were negative for GINS2, $93(60.8 \%)$ were weakly positive for GINS2 expression, $25(16.3 \%)$ were positive for GINS2 expression and $6(3.9 \%)$ were strongly positive for GINS2 expression (Figure 2E).

\section{Knockdown of GINS2 suppressed lung cancer cell proliferation, growth and colony formation in vitro}

We assessed the expression level of GINS2 via qRT-PCR. The results showed that GINS2 mRNA was highly expressed in A549 cells, moderately expressed in 95D, NCI-H1975 cells and NCI-H1299, and lowly expressed in HEK293 and HUVECs cells (Figure 3). A549 cells were selected and used for subsequent experiments.
A

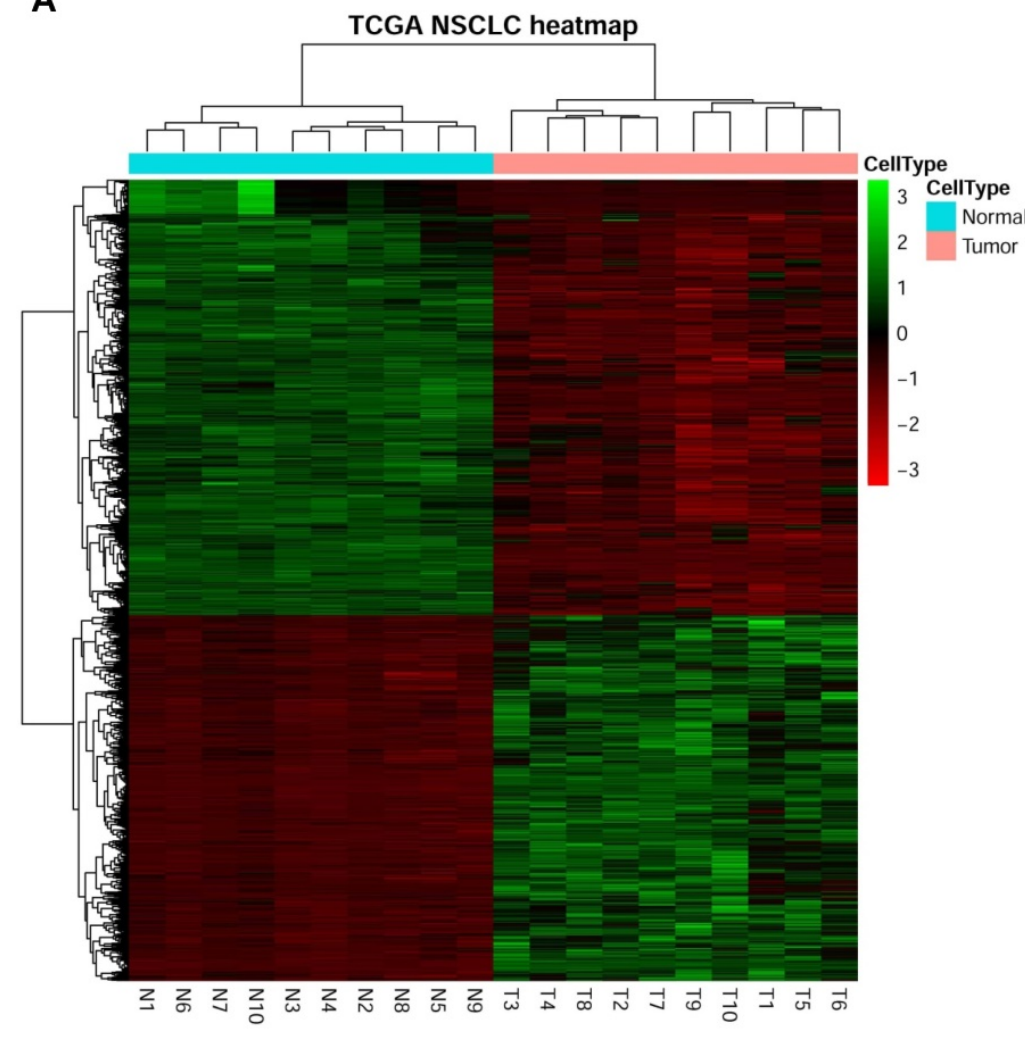

B

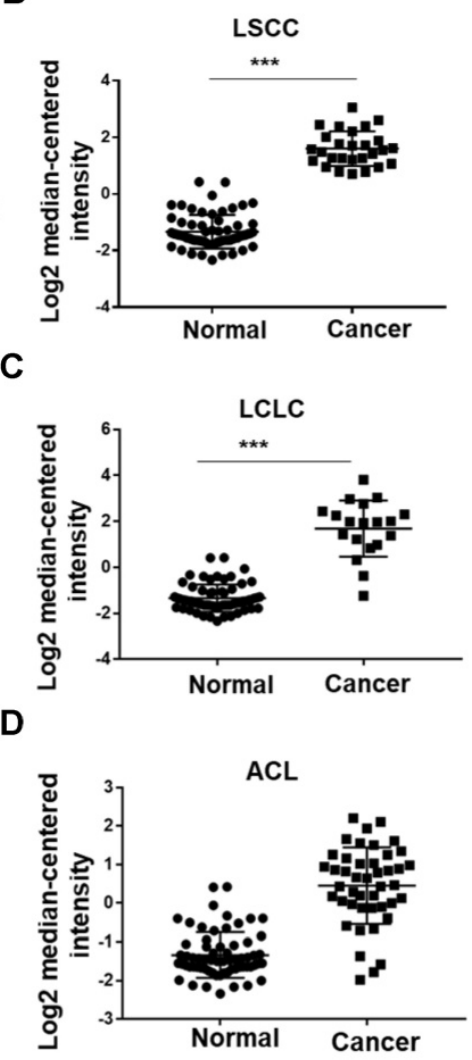

Figure 1. GINS2 was overexpressed in non-small-cell lung cancer (NSCLC) in the Cancer Genome Atlas database (TCGA). (A) Heat map from the TCGA database showing upand downregulation of selected NSCLC-related genes. (B) (C) (D) GINS2 mRNA expression was measured in lung squamous cell carcinoma (LSCC), large cell lung carcinoma $(\mathrm{LCLC})$ and adenocarcinoma of the lung $(\mathrm{ACL})$ compared to paracancerous tissue $(\mathrm{PCT})$. Data are expressed as the mean $\pm \mathrm{SEM}, \mathrm{n}=89$. $* * * P<0.001$ vs. control. 
A

B
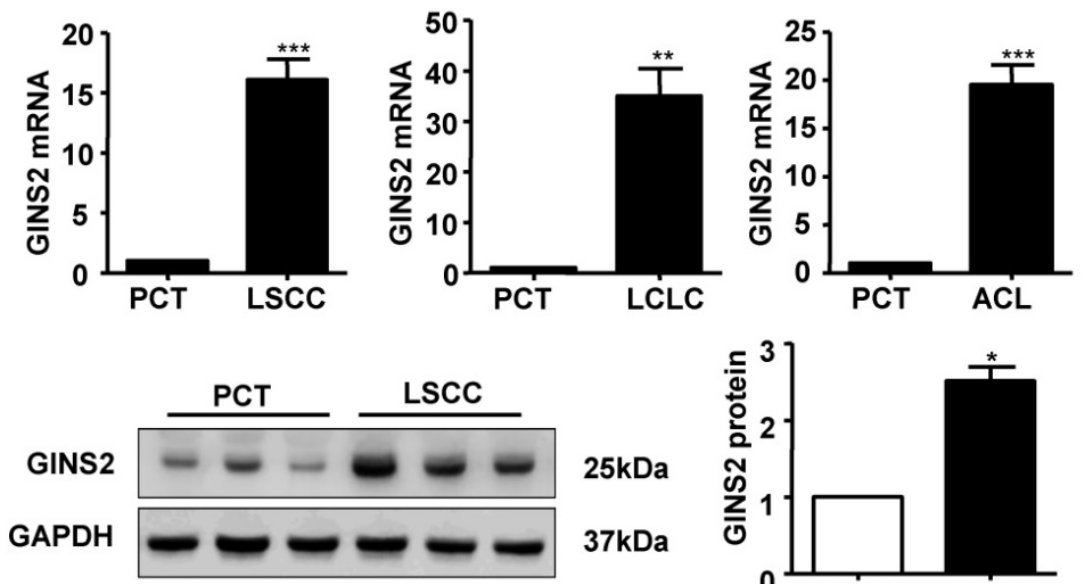

$25 \mathrm{kDa}$

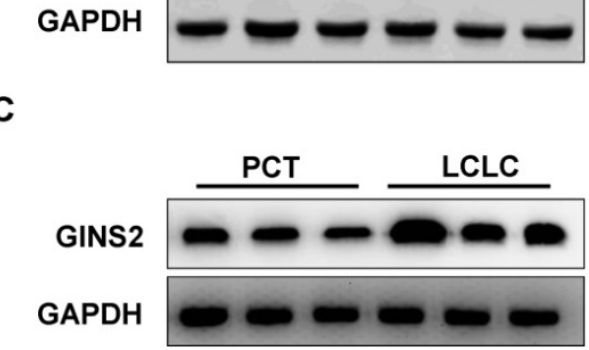

$37 \mathrm{kDa}$

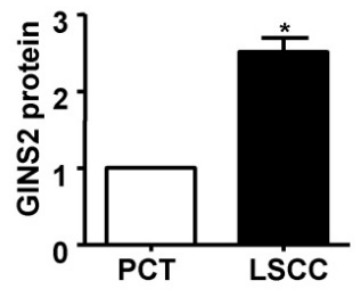

$25 \mathrm{kDa}$

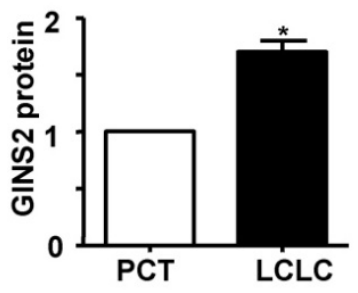

D

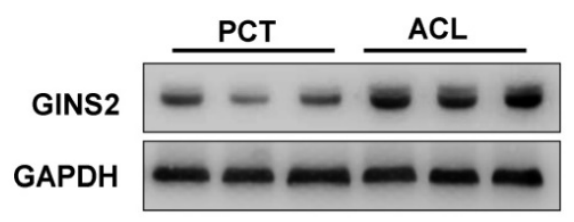

$25 \mathrm{kDa}$

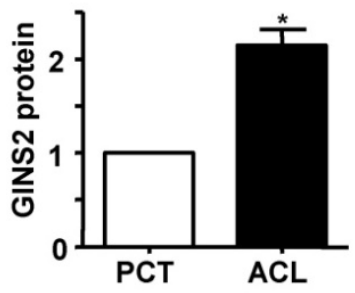

E
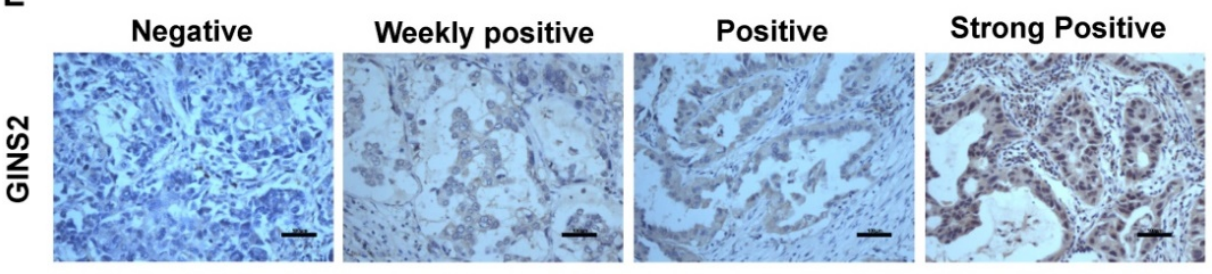

Figure 2. GINS2 was overexpressed in NSCLC tissues compared with PCTs. (A) GINS2 mRNA expression was determined by RT-PCR in LSCC, LCLC and ACL tissues and compared to that in PCTs. (B) (C) (D) Protein expression of GINS2 was determined by Western blotting in LSCC, LCLC and ACL compared to PCT. GAPDH was used as a loading control. Samples were pooled from 6 patients in each of the indicated groups. (E) GINS2 protein expression was measured by immunohistochemical staining. Data were expressed as the mean $\pm S E M, n=6 . * P<0.05, * * P<0.01, * * * P<0.001$ vs. PCT.

Second, we knocked down GINS2 gene expression in A549 cells. In order to evaluate the transfection efficiency of shCtrl and shGINS2, we observed the morphological characteristic of GFP protein in A549 cells after transfection of $72 \mathrm{~h}$ by inverted fluorescence microscope. The results showed that the transfection efficiency reached $70 \%$ after $72 \mathrm{~h}$ (Figure 4A). In addition, the mRNA and protein expression levels of GINS2 in the shGINS2 group were significantly lower than those in the shCtrl group (Figure 4B, C and D).

We counted the cells every 5 days in the shCtrl and shGINS2 groups to detect cell growth. We found that knockdown of GINS2 significantly inhibited the cell growth ratio (Figure 4E and F). Second, we measured the cell proliferation of shGINS2 cells using the MTT assay. As shown in Figure 4G, after silencing GINS2 in A549 cells, the viable cell number increased significantly compared with that in the shCtrl group on days 4 and 5. Hence, knockdown of GINS2 inhibited cell proliferation (Figure 4G). Furthermore, we detected cell growth after transfection of shGINS2 and shCtrl lentivirus using a colony formation assay (Figure 4H).

\section{Knockdown of GINS2 induced cell cycle arrest, apoptosis and migration}

To further understand the function of GINS2 in the cell cycle and apoptosis, fluorescence-activated cell sorting was used to detect the cell cycle and 
apoptosis rate. As shown in Figure 5A, the apoptosis rate was significantly higher in the shGINS2 group $(8.38 \pm 0.29 \%)$ than in the control group $(4.41 \pm 0.38 \%)$. Furthermore, after transfection with shGINS2 for 5 days, the percentages of A549 lung cancer cells in G2/M-phase and S-phase were significantly decreased, while the percentage of cells in G1-phase was dramatically increased (Figure 5B). These results indicated that cells with silenced GINS2 expression had a G2/M phase block.

Additionally, Bcl-2 protein expression was significantly decreased in the shGINS2 group compared with the control group. Bax and caspase-3 protein expression was significantly increased in the shGINS2 group compared with the control group (Figure 5C). On the other hand, CDKN1A and CDKN1B protein expression were significantly upregulated in the shGINS2 group compared to the control group (Figure 5D). CDK4 and cyclin D protein expression was decreased significantly in the shGINS2 group compared to the control group (Figure 5D).

A

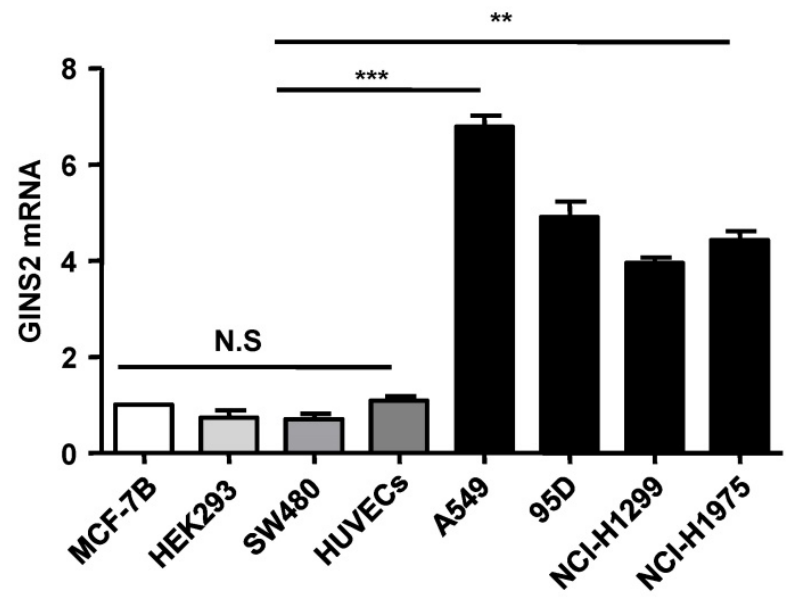

B

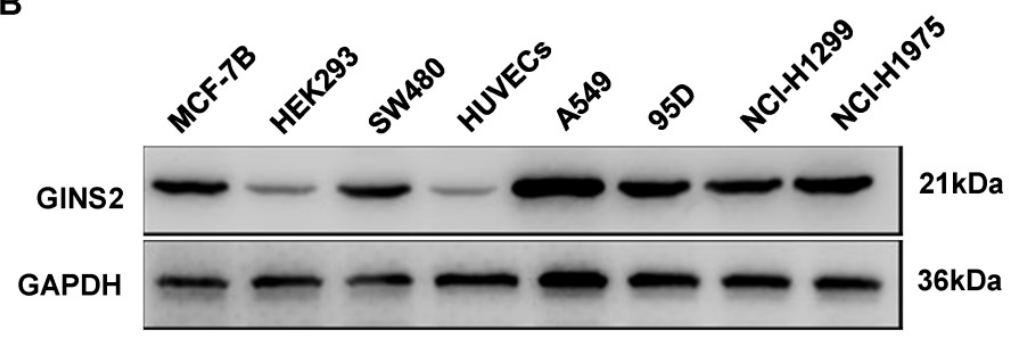

C

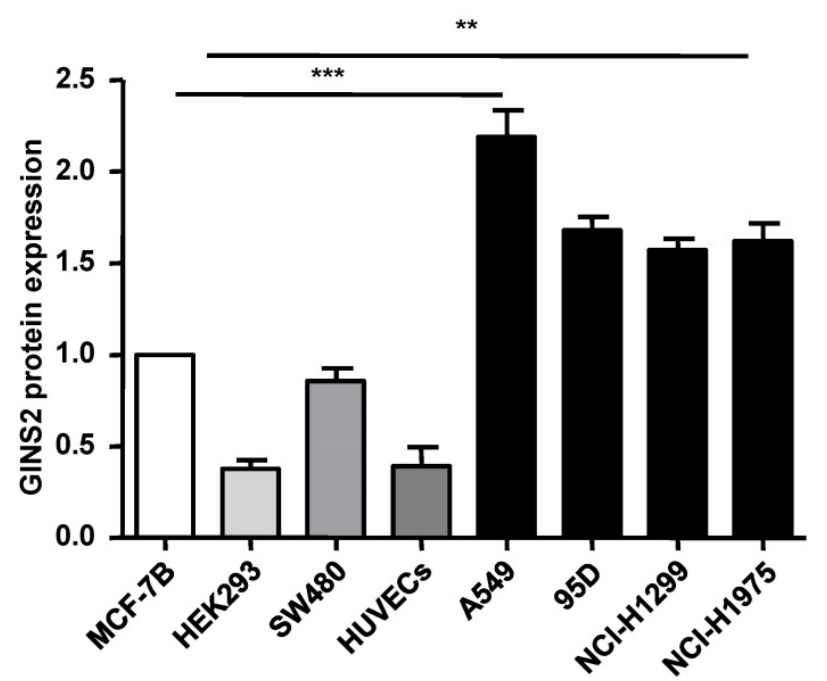

Figure 3. The expression pattern of GINS2 in cell lines. (A) GINS2 mRNA expression was measured by RT-PCR. (B) GINS2 protein expression was determined by Western blotting. (C) A representative blot and quantitation of data from six independent experiments were shown (GAPDH was used as loading control). The results were expressed as fold change compared with the NC cells. Data were expressed as the mean $\pm S E M, * P<0.05, * * P<0.01$ vs. shCtrl. 

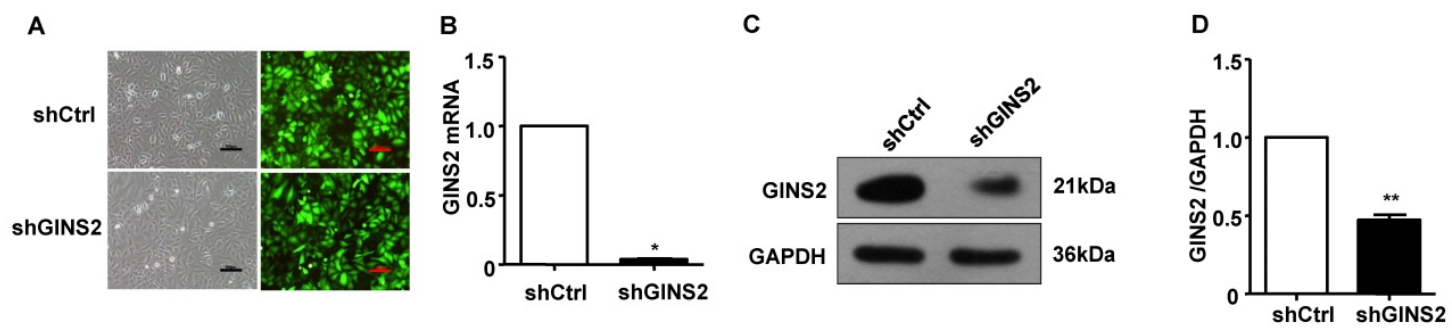

E

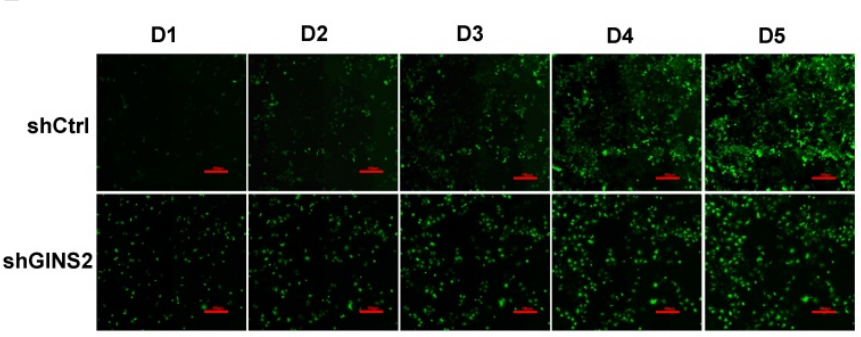

$\mathbf{F}$

G

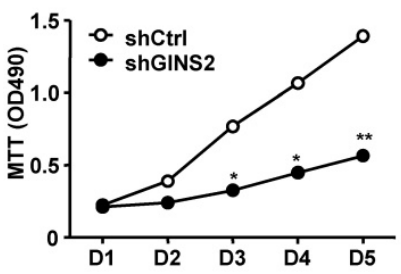

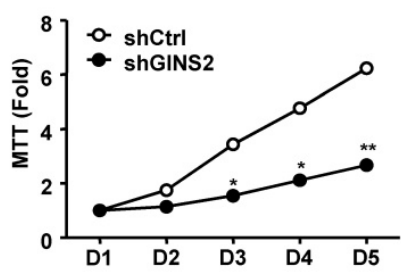
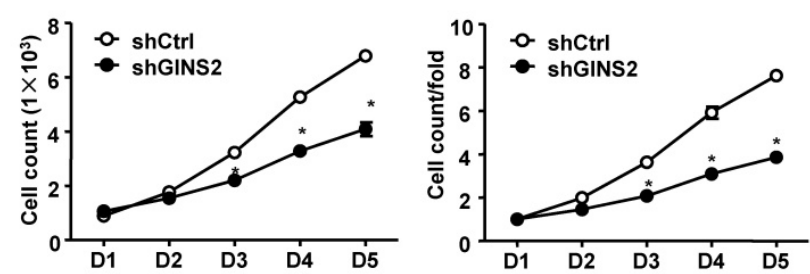

H
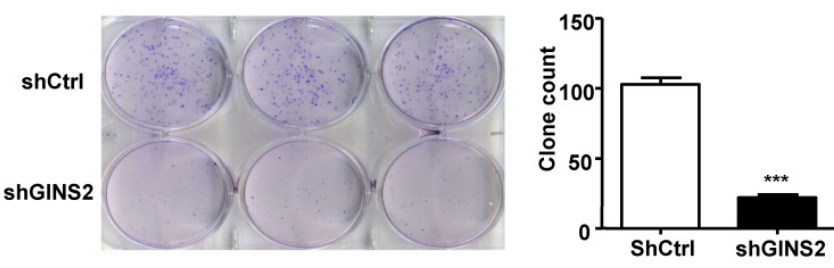

Figure 4. Knockdown of GINS2 inhibited A549 cell proliferation. (A) Fluorescence images of cells before and after transfection $(\times 100)$. (B) GINS2 mRNA expression was significantly lower in the shGINS2 group than in the control group. (C) GINS2 protein expression was significantly lower in the shGINS2 group than in the control group. (D) A representative blot and quantitation of data from six independent experiments were shown (GAPDH was used as loading control). (E) A549 cell growth was measured every day for 5 days. Fluorescence images of cells were collected every day $(\times 100)$. (F) A growth curve was generated to demonstrate the A549 cell growth rate in the shGINS2 and control groups. (G) The proliferation of A549 cells was significantly inhibited in the shGINS2 group compared to the control group. (H) GINS2 knockdown significantly reduced colony formation in $\mathrm{A} 549$ cells, as assessed by colony formation assay. Data were expressed as the mean $\pm \mathrm{SEM}, \mathrm{n}=6$. $* P<0.05, * * P<0.01$ vs. shCtrl.

In addition, we performed the Transwell assay and found that knockdown of GINS2 inhibited cell migration (Figure 6A). We also performed a cell wound scratch assay to assess cell mobility. We have outlined the cell boundary with dotted lines at the cell edge to clearly show the cell migration, the results showed that knockdown of GINS2 significantly reduced cell mobility (Figure 6B).

\section{Silencing of GINS2 inhibited tumorigenesis in nude mice}

We performed an experiment to explore the effect of GINS2 on tumor progression in vivo. After euthanasia of mice, we measured the volume, weight and size of the tumors. As shown in Figure 7A, the tumor size was smaller in the shGINS2 group. Moreover, the tumor volumes in the shGINS2 group were significantly reduced compared to those in the control group (Figure 7B). Meanwhile, the weight of the tumors was significantly decreased in the shGINS2 group compared to the shCtrl group (Figure 7C).

\section{Elucidation of the mechanism of GINS2 in tumorigenesis in vitro}

In our study, we found that knockdown of GINS2 in A549 cells inhibited cell proliferation and migration. To elucidate the mechanism of GINS2 in tumorigenesis in NSCLC, we performed RNA sequence analysis in A549 cells after knockdown of GINS2. We found 697 differentially expressed genes after knockdown GINS2 in A549 cells, and the results are shown as a volcano plot (Figure 8A). Then, we selected 30 genes and performed qRT-PCR to verify the RNAseq results and found that the results were consistent with the RNAseq analysis (Figure 8B, C and D).

Additionally, we performed Western blotting to measure protein expression in A549 cells after GINS2 knockdown. BMPR2, IFIT1, IRF1 and IFITM1 protein expression was not changed in the GINS2 knockdown group compared to the control group (Figure 9A, B and C). STAT1, STAT2 and TNFAIP3 protein expression were all upregulated after GINS2 knockdown (Figure 9B, C and D). 
A
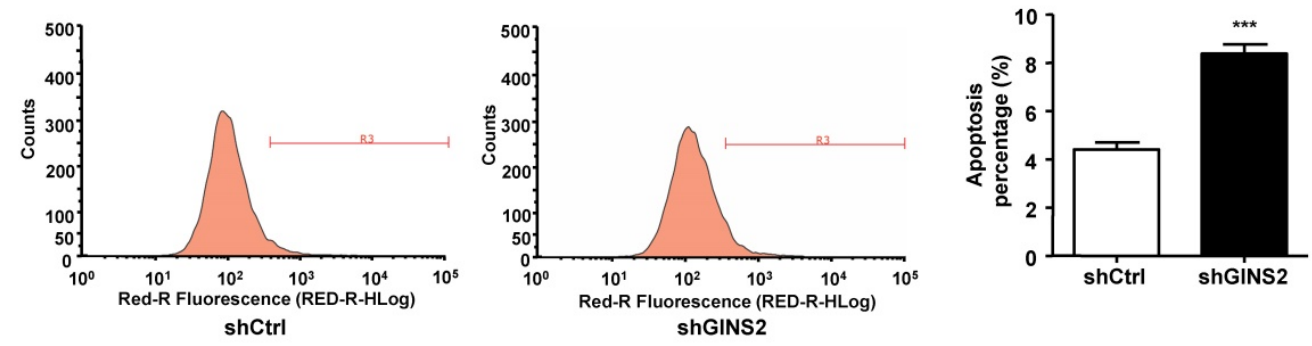

B
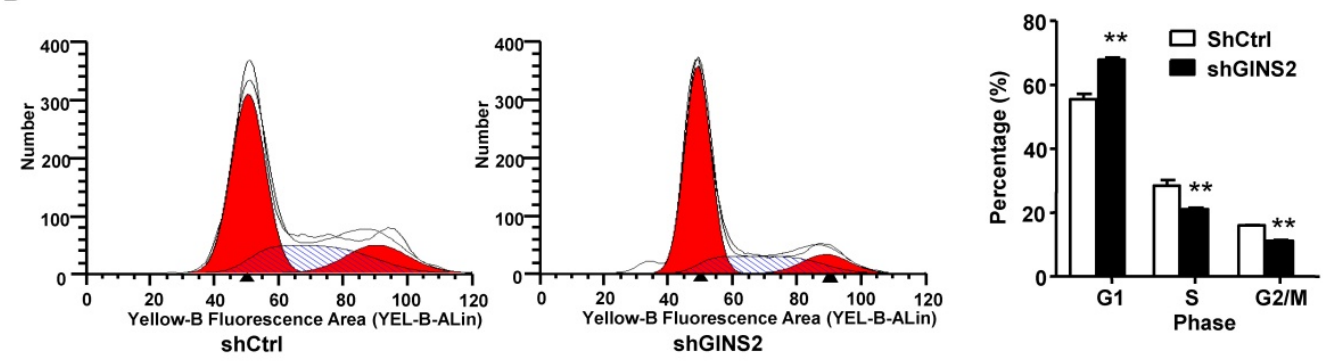

C

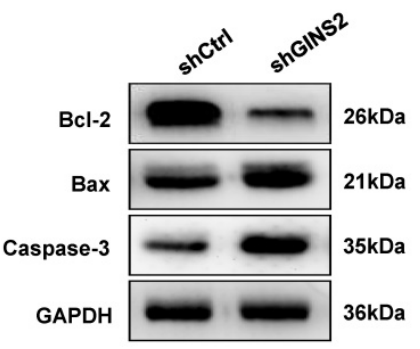

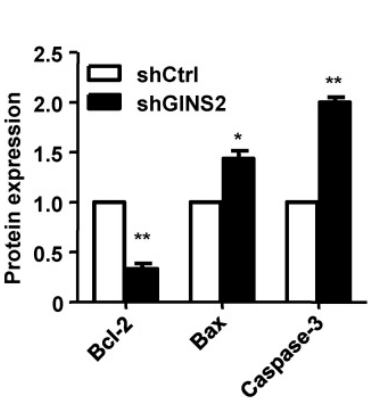

D

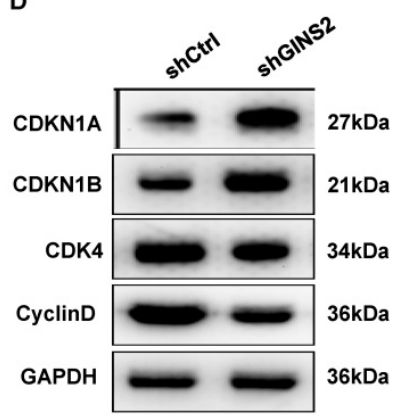

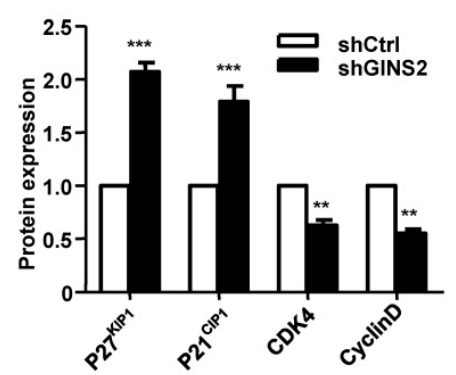

Figure 5. Knockdown of GINS2 promoted A549 cell apoptosis and proliferation stagnation. (A) A549 cells were transfected with shGINS2 and shCtrl lentivirus. (B) The proportion of cells in the G1 phase was significantly increased. (C) B-cell lymphoma-2 (Bcl-2) protein expression was increased significantly in the shGINS2 group compared to the control. Bcl-2 Associated X (Bax) and caspase-3 protein expression was decreased significantly in the shGINS2 group compared with the control. (D) Cyclin-dependent kinase inhibitor la (CDKIIA) and cyclin-dependent kinase inhibitor lb (CDKIIB) protein expression was higher in shGINS2 cells than in control cells. Cyclin-dependent kinase 4 (CDK4) and cyclin D protein expression was decreased in shGINS2 cells compared with control cells. GAPDH was used as a loading control. Samples were pooled from 6 patients in each of the indicated groups. Data were expressed as the mean $\pm S E M, n=6 . * P<0.05, * * P<0.01, * * * P<0.001 \mathrm{vs}$. shCtrl.

\section{Discussion}

This study combined TCGA database analysis and an animal model to investigate the role of GINS2 in NSCLC progression. In the TCGA database analysis, a significant correlation between increased GINS2 expression and carcinogenesis of lung cancer was observed. In addition, functional studies revealed that GINS2 promoted cell migration and proliferation and that STAT1 and STAT2 protein expression was elevated with GINS2 knockdown. Strikingly, the results showed the harmful effects of GINS2 in NSCLC progression in vivo. Moreover, RNA sequence analysis demonstrated that silencing GINS2 upregulated STAT1 and STAT2 protein expression in A549 cells. Hence, the expression level of GINS2 can influence physiological changes in NSCLC though the STAT signaling pathway.
Increased expression levels of GINS2 have been reported in many types of cancer, including thyroid cancer [20], epithelial ovarian cancer [21], cervical cancer [22], breast cancer [7, 23] and chronic myelogenous leukemia [11]. In thyroid and epithelial ovarian cancer, GINS2 plays an essential role in cell apoptosis and proliferation and may be a potential biomarker for the diagnosis or prognosis of patients in the future [20, 21]. Similarly, patients with higher GINS2 expression had shorter overall survival than patients with low GINS2 expression in early-stage cervical cancer [22]. Previous studies considered the potential effects of GINS2 on cancer progression. Here, our results from TCGA data analysis confirmed the association between increased GINS2 expression and NSCLC progression. Additionally, the increased expression of GINS2 in tumor tissues observed in the NSCLC cohort supported the crucial role of GINS2 in NSCLC development. 
A
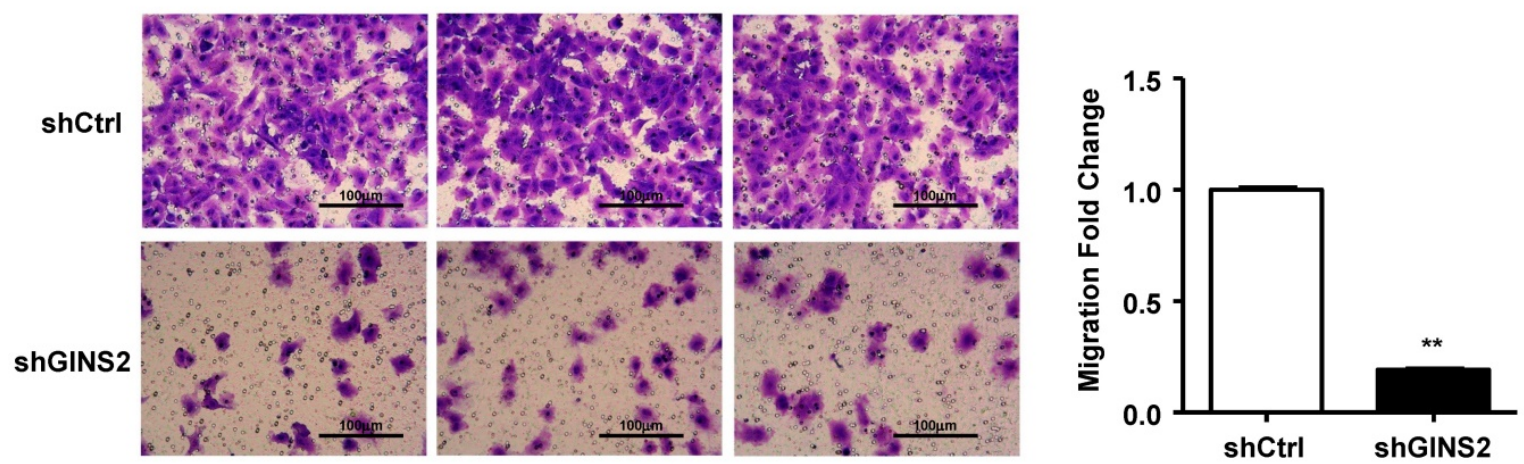

B
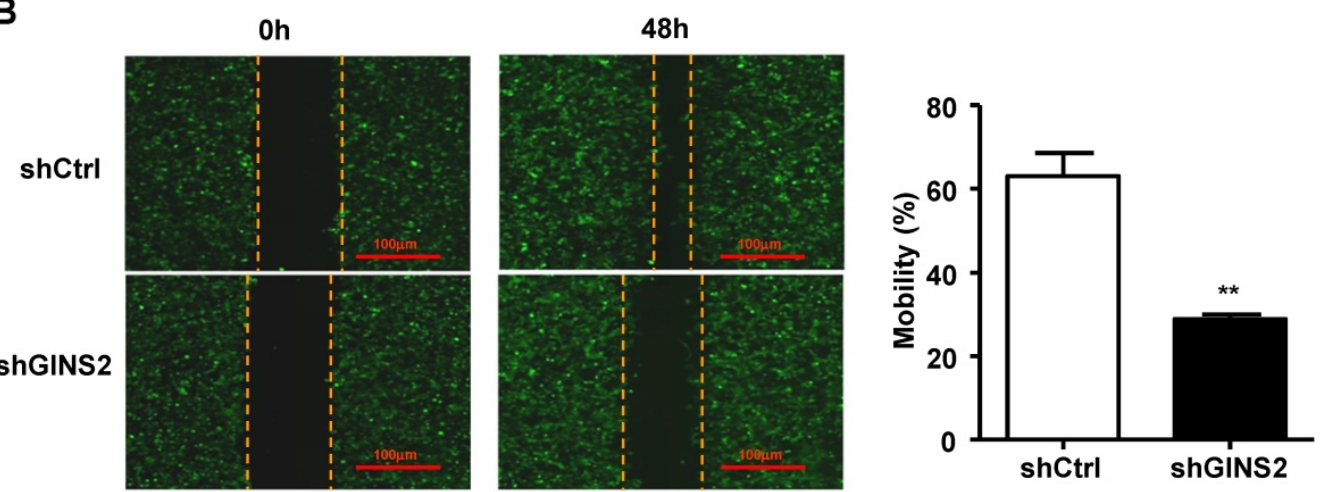

Figure 6. Knockdown of GINS2 inhibited the invasion of A549 cells. (A) A549 cells were analyzed by Transwell assay. (B) The cell scratch test was used to test cell mobility. Experiments were all performed in triplicate. Data were expressed as the mean $\pm \mathrm{SEM}, * * P<0.01$, vs. shCtrl.

A

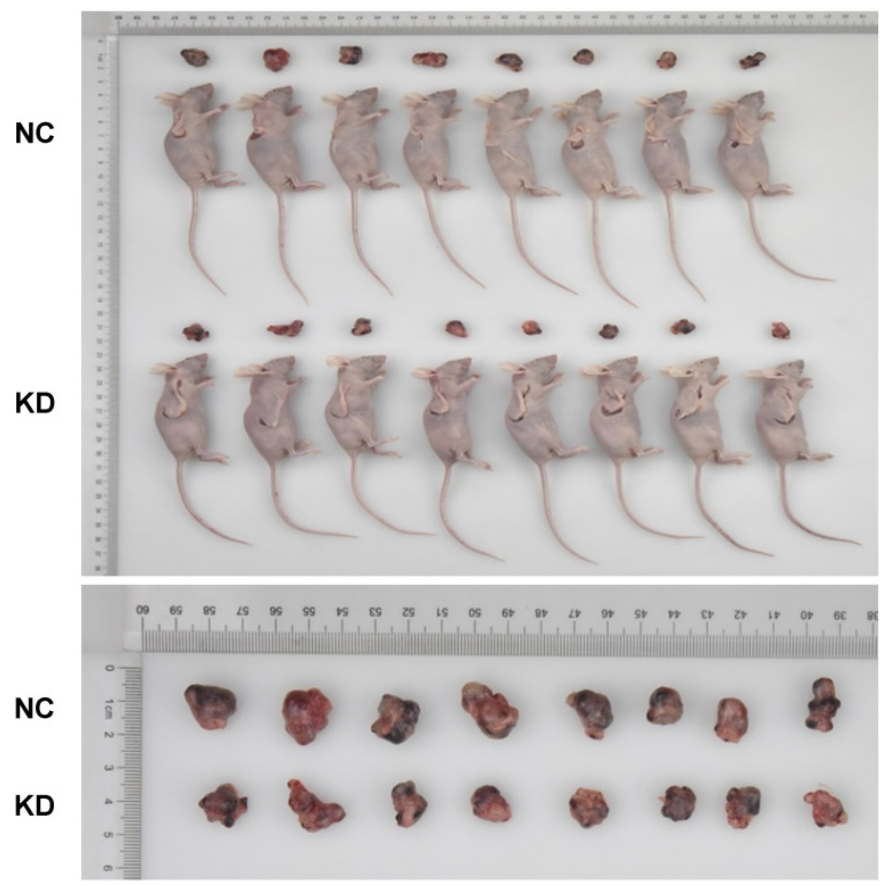

NC: 2, 5, 3, 4, 6, 10, $1,9$.

left to right

KD: $15,17,18,16,19,14,13,11$.

left to right
B
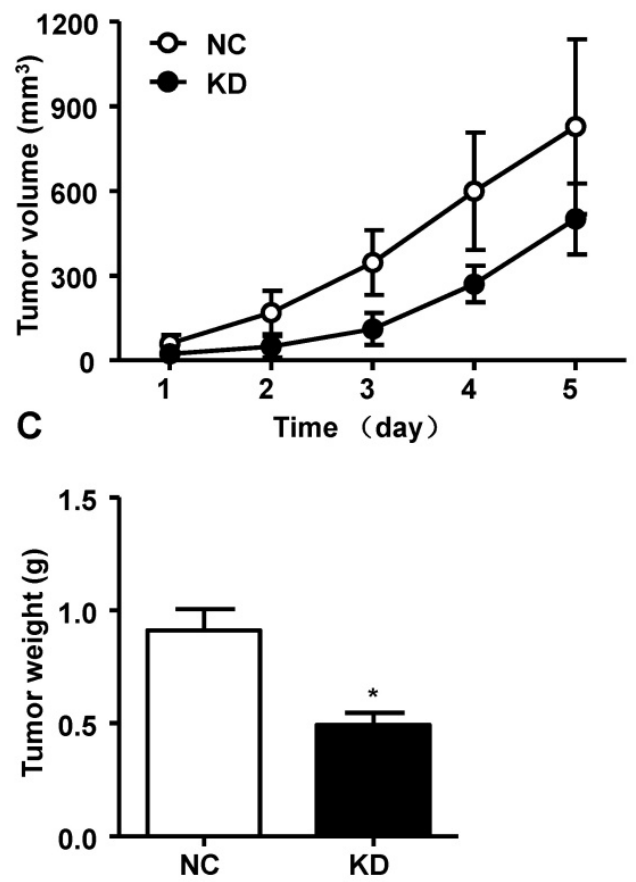

Figure 7. The effect of GINS2 knockdown on tumor progression in vivo. (A) The subcutaneous xenograft mouse models and representative images of tumors in mice. (B) Changes in tumor volume. (C) Changes in the tumor weight. NC, negative control. KD, knockdown. Data were expressed as the mean $\pm S E M$, $* * P<0.01$, vs. shCtrl. 
A

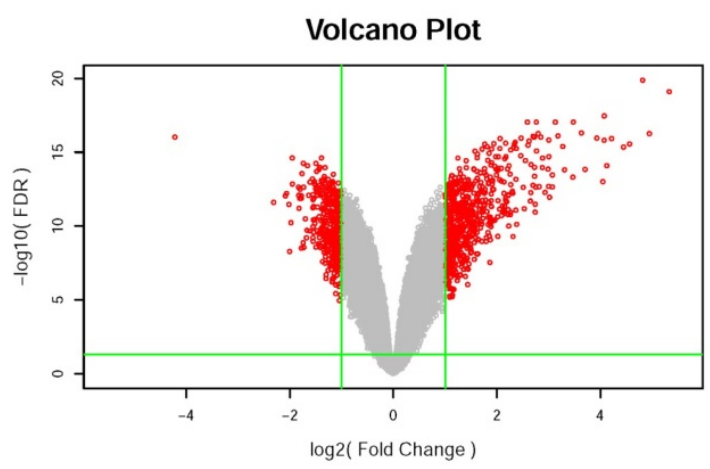

C

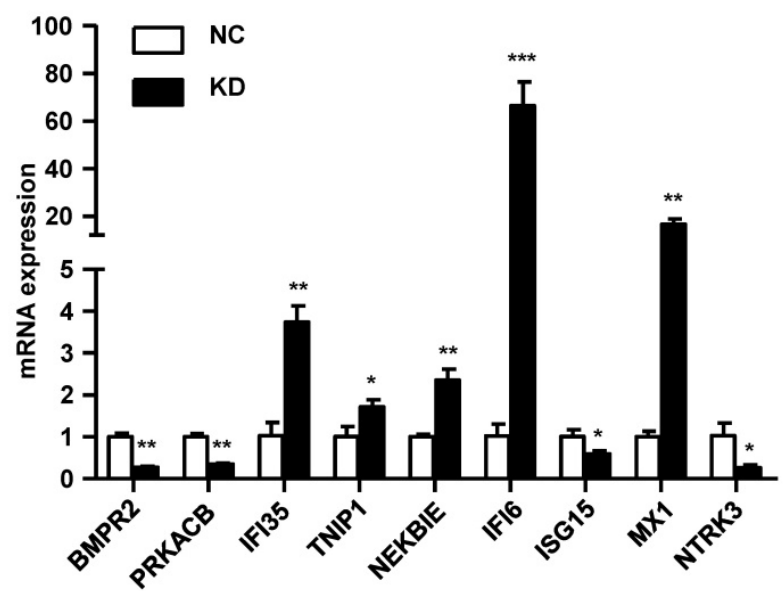

B

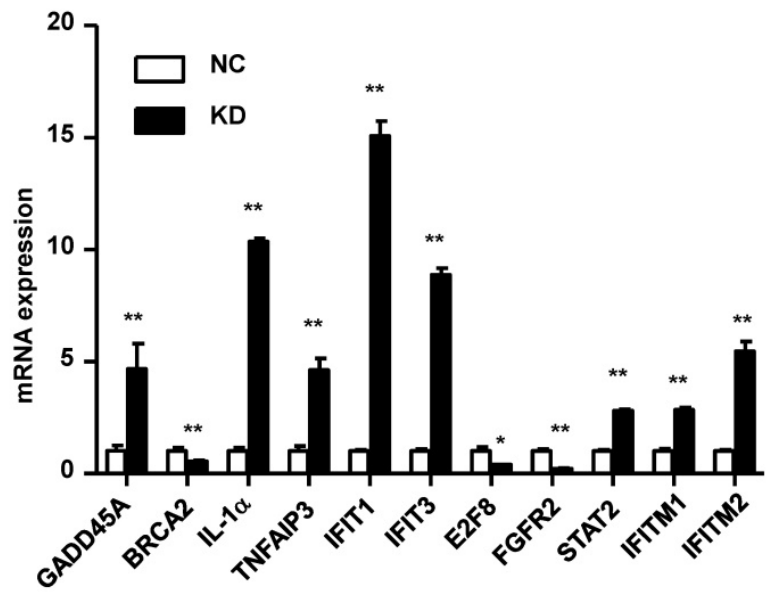

D

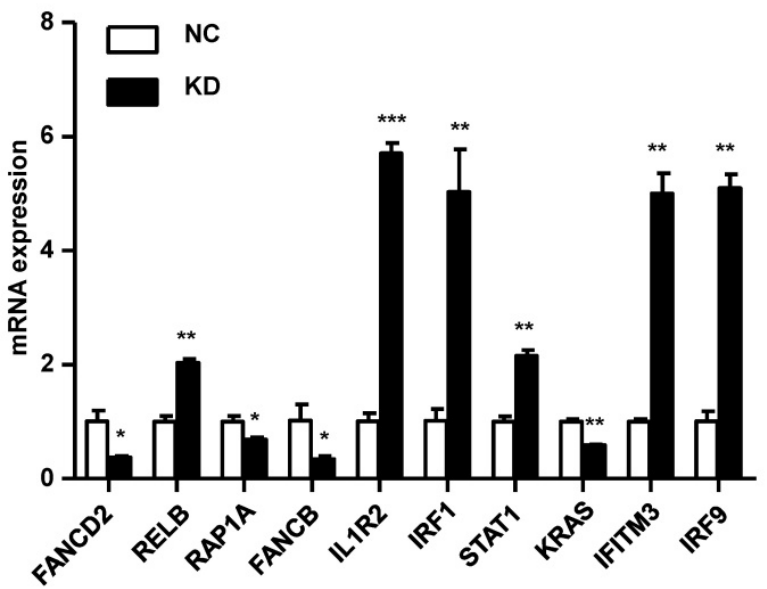

Figure 8. Mechanistic study of GINS2 knockdown in A549 cells. (A) Differentially expressed genes are shown as a volcano plot. (B) (C) (D) mRNA expression was assessed by RT-PCR. Data were expressed as the mean \pm SEM, $* * P<0.01$, vs. shCtrl.

We initially detected GINS2 mRNA expression in human lung cancer cell lines. Our results showed that A549 cells expressed high levels of GINS2, whereas 95D and NCI-H1975 cells expressed moderate levels. The reason for this difference may be the heterogeneity of cancer cells [24]. In subsequent studies, we selected A549 cells to evaluate the effect of GINS2 and to determine its function in lung cancer.

The expression levels of apoptosis-related genes Bax and Bcl-2 were altered by GINS2 in a previous study, and these findings were consistent with ours [11]. Tumorigenesis assays showed that the volume, size and weight of tumors in the shGINS2 group were significantly decreased compared to those in the control group, demonstrating that GINS2 knockdown inhibited tumor proliferation and growth in vivo.

To reveal the molecular mechanisms of GINS2 in NSCLC, we performed RNA sequencing to identify differentially expressed genes after GINS2 knockdown in A549 cells. Analysis of the cell signaling pathway showed that silencing GINS2 promoted STAT1 and STAT2 mRNA expression.
STAT1 functions as a stimulator of growth factors, cytokines and hormones in several cell types [25]. Previous research revealed that STAT1 acted as a tumor suppressor in esophageal squamous cell carcinoma and that constitutively activated STAT1 decreased cell growth and induced apoptosis [26]. In another study, STAT2 knockout mice formed larger tumors than wild-type mice, indicating that STAT2 reduces tumor growth in a syngeneic tumor transplantation model [27]. In addition to detecting gene expression, we found that GINS2 knockdown increased STAT1 and STAT2 protein expression and inhibited tumor migration and proliferation, which was consistent with a previous study. Furthermore, Huang et al reported that overexpression of TNFAIP3 inhibited migration and invasion in nasopharyngeal carcinoma [28]. Our results showed that GINS2 knockdown increased TNFAIP3 protein expression, suggesting that TNFAIP3 may be involved in the effects of GINS2 on NSCLC proliferation and migration. 
A

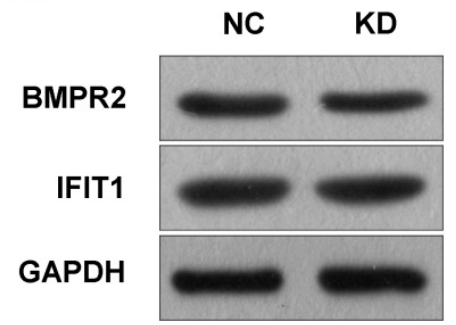

B

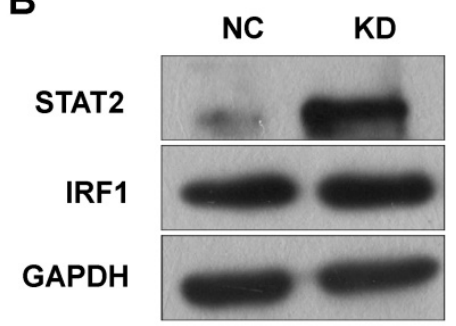

C

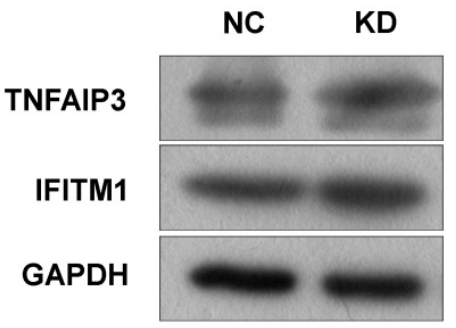

D

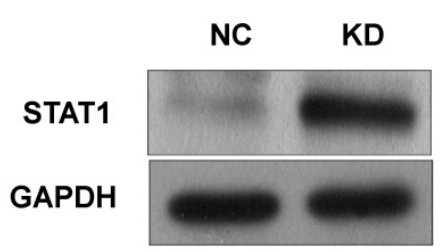

BMPR2

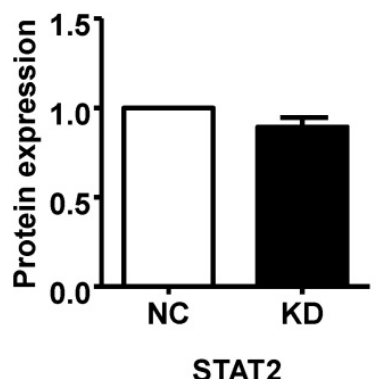

$113 \mathrm{kDa}$

$37 \mathrm{kDa}$

$36 \mathrm{kDa}$

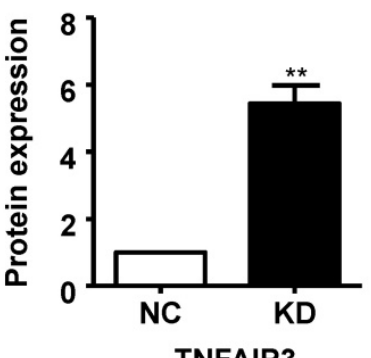

$90 \mathrm{kDa}$

$14 \mathrm{kDa}$

$36 \mathrm{kDa}$
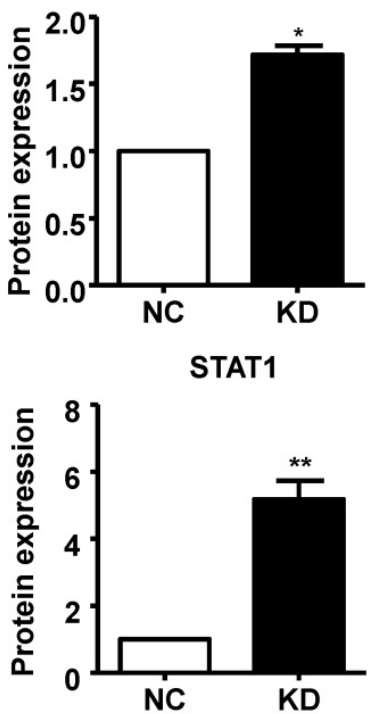

IFIT1

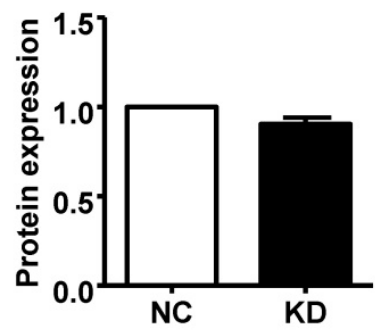

IRF-1

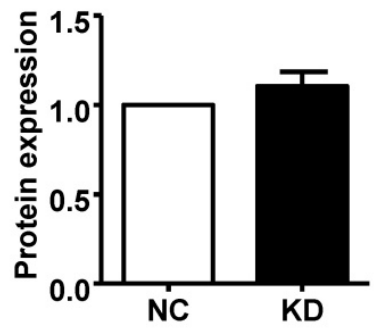

IFITM1

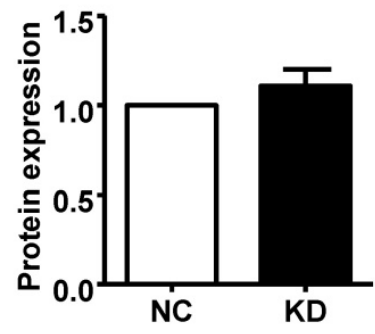

Figure 9. Knockdown of GINS2 unregulated the signal transducer and activator of transcription (STAT) signaling pathway. (A) Knockdown of GINS2 did not improve bone morphogenetic protein receptor type 2 (BMPR2) or interferon-induced protein with tetratricopeptide repeat 1 (IFIT1) protein expression. (B) Knockdown of GINS2 unregulated STAT1 protein expression. (C) Knockdown of GINS2 upregulated TNF alpha-induced protein 3 (TNFAIP3) protein expression. (D) Knockdown of GINS2 unregulated STAT1 protein expression. Experiments in this figure were all performed in triplicate. Data were expressed as the mean \pm SEM, $* * P<0.01$, vs. shCtrl.

Taken together, based on previous studies, we determined the clinical significance of GINS2 in NSCLC and demonstrated that GINS2 knockout significantly impaired the proliferation and tumorigenicity of A549 cells. Furthermore, GINS2 inhibits the proliferation of NSCLC by inhibiting the STAT signaling pathway, which may be a potential biomarker for the diagnosis or prognosis of lung cancer.

\section{Acknowledgements}

This study was supported by grants from the Natural Science Foundation of Shandong Province (NO.ZR2016HM78), the Key Research and Development Program of Shandong Province
(NO.2018GSF118221), and the National Natural Science Foundation of China Youth Found (NO.81602031).

\section{Abbreviations}

Abs: antibodies; ACL: adenocarcinoma of the lung; ATCC: American Type Culture Collection; Bcl-2: b-cell lymphoma-2; BMPR2: bone morphogenetic protein receptor type 2; CDKN1A: cyclin dependent kinase inhibitor 1A; CDK4: cyclin dependent kinases 4; DMEM: Dulbecco's modified Eagle's medium; FDR: false discovery rate; FBS: fetal bovine serum; GAPDH: glyceraldehyde-3-phosphate dehydrogenase; GINS: GINS complex subunit; HEK293: human embryonic kidney 293; HUVECs: human umbilical vein 
endothelial cell; IFIT1: interferon induced protein with tetratricopeptide repeats 1; IFITM1: interferon induced transmembrane protein 1; IRF-1: interferon regulatory factor 1; KD: knock down; LCLC: large cell lung cancer; LSCC: lung squamous cell carcinoma; NC: negative control; NSCLC: non-small cell lung cancer; PBS: phosphate-buffered saline; PVDF: polyvinylidene difluoride; PCT: para-carcinoma tissue; RNA-Seq: RNA sequencing; STAT: signal transducer and activator of transcription; TCGA: The Cancer Genome Atlas; TNFAIP3: tumor necrosis factor alpha induced protein 3 .

\section{Supplementary Material}

Supplementary tables.

http://www.jcancer.org/v12p0099s1.pdf

\section{Competing Interests}

The authors have declared that no competing interest exists.

\section{References}

1. Chen W, Zheng R, Zeng H, Zhang S. Epidemiology of lung cancer in China [J]. Thorac Cancer. 2015; 6: 209-15.

2. Torre LA, Bray F, Siegel RL, Ferlay J, Lortet-Tieulent J, Jemal A. Global cancer statistics, 2012 [J]. CA Cancer J Clin. 2015; 65: 87-108.

3. Ryska A, Berzinec P, Brcic L, Cufer T, Dziadziuszko R, Gottfried M, et al. NSCLC molecular testing in Central and Eastern European countries [J]. BMC Cancer. 2018; 18: 269

4. Fledelius J, Winther-Larsen A, Khalil AA, Hjorthaug K, Frokiaer J, Meldgaard P. Assessment of very early response evaluation with (18)F-FDG-PET/CT predicts survival in erlotinib treated NSCLC patients-A comparison of methods [J]. Am J Nucl Med Mol Imaging. 2018; 8: 50-61.

5. Gourd E. Durvalumab boosts progression-free survival in NSCLC [J]. Lancet Oncol. 2018; 19: e11.

6. Gambus A, Jones RC, Sanchez-Diaz A, Kanemaki M, van Deursen F, Edmondson RD, et al. GINS maintains association of Cdc45 with MCM in replisome progression complexes at eukaryotic DNA replication forks [J]. Nat Cell Biol. 2006; 8: 358-66.

7. Rantala JK, Edgren H, Lehtinen L, Wolf M, Kleivi K, Vollan HK, et al. Integrative functional genomics analysis of sustained polyploidy phenotypes in breast cancer cells identifies an oncogenic profile for GINS2 [J]. Neoplasia. 2010; 12 : 877-88

8. Stamova BS, Apperson M, Walker WL, Tian Y, Xu H, Adamczy P, et al. Identification and validation of suitable endogenous reference genes for gene expression studies in human peripheral blood [J]. BMC Med Genomics. 2009; 2: 49 .

9. Lee $\mathrm{SH}, \mathrm{Nam} J \mathrm{~K}$, Park JK, Lee JH, Min do S, Kuh HJ. Differential protein expression and novel biomarkers related to $5-\mathrm{FU}$ resistance in a $3 \mathrm{D}$ colorectal adenocarcinoma model [J]. Oncol Rep. 2014; 32: 1427-34.

10. Zheng M, Zhou Y, Yang X, Tang J, Wei D, Zhang Y, et al. High GINS2 transcript level predicts poor prognosis and correlates with high histological grade and endocrine therapy resistance through mammary cancer stem cells in breast cancer patients [J]. Breast Cancer Res Treat. 2014; 148: 423-36.

11. Zhang X, Zhong L, Liu BZ, Gao YJ, Gao YM, Hu XX. Effect of GINS2 on proliferation and apoptosis in leukemic cell line [J]. Int J Med Sci. 2013; 10: 1795-804.

12. Chi F, Wang Z, Li YZ, Chang N. Knockdown of GINS2 inhibits proliferation and promotes apoptosis through the p53/GADD45A pathway in non-small-cell lung cancer [J]. Bioscience Reports. 2020; 40: BSR20193949.

13. Liu $X L$, Sun $L$, Zhang $S$, Zhang SG, Li WY. GINS2 facilitates epithelial-to-mesenchymal transition in non-small-cell lung cancer through modulating PI3K/Akt and MEK/ERK signaling [J]. Journal of Cellular Physiology 2019; [Epub ahead of print].

14. Albrecht C, Hosiner S, Tichy B, Aldrian S, Hajdu S, Nurnberger S. Comparison of Lentiviral packaging mixes and producer cell lines for RNAi applications [J]. Mol Biotechnol. 2015; 57: 499-505.

15. Jin H, Xu G, Zhang Q, Pang Q, Fang M. Synaptotagmin-7 is overexpressed in hepatocellular carcinoma and regulates hepatocellular carcinoma cell proliferation via Chk1-p53 signaling [J]. Onco Targets Ther. 2017; 10: 4283-93.

16. Wang W, Chen Y, Bai L, Zhao S, Wang R, Liu B, et al. Transcriptomic analysis of the liver of cholesterol-fed rabbits reveals altered hepatic lipid metabolism and inflammatory response [J]. Sci Rep. 2018; 8: 6437.
17. Guan H, Lin Y, Bai L, An Y, Shang J, Wang Z, et al. Dietary cocoa powder improves hyperlipidemia and reduces atherosclerosis in apoE deficient mice through the inhibition of hepatic endoplasmic reticulum stress [J]. Mediator of Inflammation. 2016; 2016: 1937572.

18. Gao S, Xu L, Zhang Y, Yu Q, Li J, Guan H, et al. Salusin-a inhibits proliferation and migration of vascular smooth muscle cell via Akt/mTOR signaling [J]. cellular Physiology and Biochemistry. 2018; 50:1740-53.

19. Han SS, Kim WJ, Hong Y, Hong SH, Lee SJ, Ryu DR, et al. RNA sequencing identifies novel markers of non-small cell lung cancer [J]. Lung Cancer. 2014; 84: 229-35.

20. Ye Y, Song YN, He SF, Zhuang JH, Wang GY, Xia W. GINS2 promotes cell proliferation and inhibits cell apoptosis in thyroid cancer by regulating CITED2 and LOXL2 [J]. Cancer Gene Ther. 2019; 26: 103-13.

21. Yan T, Liang W, Jiang E, Ye A, Wu Q, Xi M. GINS2 regulates cell proliferation and apoptosis in human epithelial ovarian cancer [J]. Oncol Lett. 2018; 16: 2591-8.

22. Ouyang F, Liu J, Xia M, Lin C, Wu X, Ye L, et al. GINS2 is a novel prognostic biomarker and promotes tumor progression in early-stage cervical cancer [J]. Oncol Rep. 2017; 37: 2652-62.

23. Peng L, Song Z, Chen D, Linghu R, Wang $Y$, Zhang X, et al. GINS2 regulates matrix metallopeptidase 9 expression and cancer stem cell property in human triple negative Breast cancer [J]. Biomed Pharmacother. 2016; 84: 1568-74.

24. Suresh PK. Tumor Heterogeneity: An important determinant for efficacy and safety in nanoparticle anticancer gene therapy [J]. Trends Biotechnol. 2018; 36:476-7.

25. Meissl K, Macho-Maschler S, Muller M, Strobl B. The good and the bad faces of STAT1 in solid tumours [J]. Cytokine. 2017; 89: 12-20.

26. Zhang Y, Molavi O, Su M, Lai R. The clinical and biological significance of STAT1 in esophageal squamous cell carcinoma [J]. BMC Cancer. 2014; 14: 791.

27. Yue C, Xu J, Tan Estioko MD, Kotredes KP, Lopez-Otalora Y, Hilliard BA, et al. Host STAT2/type I interferon axis controls tumor growth [J]. Int J Cancer. 2015; 136: 117-26.

28. Huang T, Yin L, Wu J, Gu JJ, Ding K, Zhang N, et al. TNFAIP3 inhibits migration and invasion in nasopharyngeal carcinoma by suppressing epithelial mesenchymal transition [J]. Neoplasma. 2017; 64: 389-94. 